\title{
DECODING PHASE-BASED INFORMATION FROM SSVEP RECORDINGS: A COMPARATIVE STUDY
}

\author{
Nikolay V. Manyakov, Nikolay Chumerin, Adrien Combaz, Arne Robben, \\ Marijn van Vliet, Marc M. Van Hulle
}

Laboratory for Neurofysiology, K.U.Leuven, Herestraat 49, bus 1021, 3000 Leuven, Belgium

\begin{abstract}
In this paper, we report on the decoding of phase-based information, from steady-state visual evoked potential (SSVEP) recordings, by means of different classifiers. In addition to the ones reported in the literature, we also consider other types of classifiers such as the multilayer feedforward neural network based on multi-valued neurons (MLMVN), and the classifier based on fuzzy logic, which we especially tuned for phasebased SSVEP decoding. The dependency of the decoding accuracy on the number of targets and on the decoding window size are discussed. When comparing existing phase-based SSVEP decoding methods with the proposed ones, we are able to show that the latter ones perform better, for different parameter settings, but especially when having multiple targets. The necessity of optimizing the target frequencies to the individual subject is also discussed.
\end{abstract}

Index Terms - Steady state visual evoked potential, phase shift, decoding, brain signals

\section{INTRODUCTION}

With a brain-computer interface (BCI), brain activity is read and used for enabling to subject to interact with the external world, without involving any muscular activity or peripheral nerves. The field has witnessed a tremendous development in recent years (see, for example, the editorial in IEEE Signal Processing Magazine [1]), and is now widely considered one of the successful outcomes of the neurosciences. BCI applications are emerging targeted to improve the quality of life of patients suffering from amyotrophic lateral sclerosis, stroke, brain/spinal cord injury, cerebral palsy, muscular dystrophy, etc. Brain-computer interfaces are either invasive [2, 3, 4] or noninvasive $[5,6]$. The invasive ones use recordings made intracortically (local field potentials and action potentials) or

NVM is supported by the research grant GOA $10 / 019$, NC is supported by IST-2007-217077, AC and AR are supported by IWT doctoral grants, MvV is supported by G.05809, MMVH is supported by PFV/10/008, CREA/07/027, G.0588.09, IUAP P6/29, GOA 10/019, IST-2007-217077, and the King Baudouin Foundation of Belgium (SWIFT prize).

The authors wish to thank Refet Firat Yazicioglu, Tom Torfs, and Chris Van Hoof, from imec in Leuven, for providing us with the wireless EEG system and for their support. from the surface of the brain (electrocorticogram), whereas the noninvasive ones mostly employ electroencephalograms (EEGs) recorded from the subject's scalp.

Several noninvasive methods have been proposed in the literature. The one we consider in this paper is based on the steady-state visual evoked potential (SSVEP). This type of BCI relies on the psychophysiological properties of EEG brain responses recorded from the occipital area during the periodic presentation of visual stimuli (flickering stimuli). When the periodic presentation is at a sufficiently high rate ( $>6 \mathrm{~Hz}$ ), the individual transient visual responses (which are time- and phase locked to the stimulus onset) overlap and give rise to a steady state signal: the signal resonates at the stimulus rate and its multipliers [7].

Conventional SSVEP-based BCI systems [8, 9, 10, 11] rely on the increase of the EEG signal amplitude at frequencies $f, 2 f, 3 f, \ldots$ to infer that the subject is looking at a target flickering at rate $f$. Since the relevant EEG activity is always embedded into other on-going brain activity, and contaminated by (recording) noise, the detection task is not straightforward. When using a brief recording interval, it is quite likely to detect an (irrelevant) increase in the amplitude at frequency $f$. To overcome this problem, and to improve the decoding performance, several remedies have been suggested: averaging over several time intervals [8], recording over a longer period of time [9], preliminary training [10], etc. Finally, in order to enhance the functionality of the BCI application, not one but several stimulation frequencies $f_{1}, \ldots, f_{N}$ are used simultaneously. In this case, each frequency corresponds to a particular command the subject can communicate. The detection problem, therefore, becomes more complex since now one of several possible stimulation frequencies $f_{i}$ needs to be detected from the EEG.

The listed methods achieve a reasonable information transfer rate [11], however, since they rely on a computer screen (monitor) for the visual stimulation, they have some limitations: the stimulation frequencies are related to the refresh rate of the computer screen [12] (e.g., $85 \mathrm{~Hz}$, as in our case), and restricted to specific (subject-dependent) frequency bands to obtain good responses [10]; the harmonics of some stimulation frequencies could interfere with one another (and their harmonics), leading to a deterioration of the decoding 
performance [12], to name a few. These restrictions limit the number of targets in a SSVEP-based BCI. In order to have more targets, the phase of the SSVEP has been proposed as an alternative $[13,14]$ : even a single frequency could be used but with different phase lags, for encoding different targets. For example, one can perform visual stimulation using $N$ targets simultaneously flickering with the same frequency $f$, but with different time delays $\Delta t_{m}=(m-1) /(f N)$ seconds for the command $m(m=1, \ldots, N)$. Thus, by extracting phase information from the Fourier transform of the EEG signal, and by comparing it to the phase of some reference signal(s) (for example, the phase of the EEG response for a stimulus with zero phase lag [14] or the phases of the EEG responses for all possible delayed stimulations [13]), one can detect the target the subject is looking at. With such an approach, the number of possible commands can be increased by combining different frequencies with different phase shifts [13] or simply by using one (well detectable) frequency with different phase shifts [14].

In this paper, we account for the problem of accurately detecting phase shifts, using only short recording intervals, and of properly assigning class labels to the estimated phase. We investigate the effect of different classifiers for decoding up to 16 phase shifted targets. We consider classifiers that have been reported in the literature for phase-based SSVEP decoding [13, 14], and two other classifiers, the multilayer neural network, containing multi-valued neurons (MLMVN) [15], and a classifier based on fuzzy logic [16], both of which we have adapted specifically for circular data (in our case, the phase shifts and resulting classes are circular).

\section{METHODS}

\subsection{EEG experiment}

The EEG recordings have been performed with a prototype miniature wireless EEG system developed by imec ${ }^{1}$ and built around their ultra-low power 8-channel EEG amplifier chip [17]. Each one of the eight channels is recorded at a sampling rate $f_{s}^{\mathrm{EEG}}=1000 \mathrm{~Hz}$ and with a resolution of 12 bits/sample. We have used an electrode cap with large filling holes and sockets for active $\mathrm{Ag} / \mathrm{AgCl}$ electrodes (ActiCap, Brain Products). The eight electrodes were placed primarily on the occipital pole, namely at positions $\mathrm{Oz}, \mathrm{O} 1, \mathrm{O} 2, \mathrm{POz}$, PO7, PO3, PO4, PO8, according to the international 10-10 system. The reference and ground electrodes were placed on the left and right mastoids, respectively.

Four healthy subjects (all male, aged 24-34 with average age 29.5 , three righthanded, one lefthanded) participated in the experiments. The experiment consisted of observing a flashing stimulus in the center of the screen (CRT monitor, refresh rate $f_{s}^{\text {scr }}=85 \mathrm{~Hz}$ ). To produce stronger SSVEP responses [12], we have used the (monitor) frame-based visual

\footnotetext{
${ }^{1}$ http://www.imec.be
}

stimulation of period of six frames: three frames of intense (white) stimulus presentation followed by three frames without the stimulus (black). The stimulation frequency is, thus, $f=85 / 6 \approx 14.16 \mathrm{~Hz}$ close to $15 \mathrm{~Hz}$, which is reported to elicit the largest SSVEP amplitude [7]. The EEG data have been collected during sessions of two minutes of visual stimulation.

\subsection{Training and testing data}

To validate the applicability and the accuracy of the classifier, it is sufficient to limit ourselves to off-line decoding. For this assessment, we opted for a 4-fold cross-validation, namely, the recorded data have been split into four parts. One of those parts has been used for training and the rest for testing. All possible selections of data for training were considered, leading to four decoding results. Since we recorded data from one flickering stimulus having no phase shifts, we introduced phase-shifted targets (stimuli) artificially in the following way. Considering that, under normal conditions, the individual SSVEP latency is stable $[13,18]$, the delay in the stimulation (using the same frequency) would introduce the same delay in the recorded EEG signal. In this way, when introducing proper shifts in the EEG signal, the responses for different phase shifted stimulations (with fixed frequency $f$ ) can be constructed. From those constructed EEGs, we cut a number of intervals with length $T$ seconds in such a way that the starting points of those intervals are aligned to the 'stimulus onset'. As a consequence, the interval's starting points are integer number of periods apart (with a length of $1 / f \mathrm{~s}$ ). We have used this strategy to generate the training and test data. While in the training set, we allowed the constructed data to be highly overlapping (to have a bigger amount of training samples), we tried to reduce those overlaps in the test set ${ }^{2}$.

\section{CLASSIFIERS}

\subsection{Methods of Jia and co-workers [13]}

In [13], the following method was proposed. Based on the training data, the reference phases $\phi_{i, f}^{r e f}$ are estimated by averaging, over the whole training set, the Fourier coefficients (obtained by using FFT) at the stimulation frequency $f$ of each target class $i=1, \ldots N$. Here, only one optimal electrode (according to [13]) is considered. When decoding new data (from the test set), the Fourier coefficients at stimulation frequency $f$ are estimated from the same previously selected optimal electrode. This coefficient is projected onto all reference $\phi_{i, f}^{r e f}$ phases and the resulting target class $i$ is selected as the one with the maximal projected value $\rho_{i}$. If we consider not only the fundamental frequency $f$, but also its harmonics (in our case only the first harmonic), then we compute the

\footnotetext{
${ }^{2}$ As we found out later on, it did not much influence the decoding accuracy
} 
projected lengths not only of the fundamental frequency, but also of its harmonics, on the respective reference phases, and sum up the resulting projections for each target class separately, and then take the argmax of the resulting projections to infer the index of the target class.

\subsection{Methods of Lee and co-workers [14]}

In [14], the following method was proposed. The EEG signals recorded from one channel were band pass filtered in the range $[f-2, f+2] \mathrm{Hz}$, where $f$ is the stimulation frequency. Based on the training data (1 min. of recordings on a subject observing a flickering stimulus with "zero" phase lag), $\mathrm{SSVEP}_{r e f}$ is generated by averaging all epochs (EEG recordings corresponding to one period of stimulation, from one channel). The reference value $t_{r e f}[\mathrm{~s}]$ is defined, from the obtained $\mathrm{SSVEP}_{\text {ref }}$, as the latency of the maximum amplitude peak. In the decoding stage, the phase lag between SSVEP $_{\text {ref }}$ and SSVEP $_{\text {gaze }}$ is evaluated to infer the target class. This was done by cutting the SSVEP $_{\text {gaze }}$ signal into 1 period segments, and take the average, and determine in this average the latency of the maximum amplitude peak, called $t_{\text {peak }}$ [s], Next, we transform the difference $\Delta t=t_{\text {ref }}-t_{\text {peak }}$ into a phase difference $\theta=2 \pi \Delta t f$. This phase difference $\theta$ is then readjusted to the interval $[0,2 \pi)$ by adding or subtracting (if necessary) $2 \pi$. The achieved phase distance $\theta$ is compared to the expected phase delays $\theta_{i}=2 \pi(i-1) / N(i=1 \ldots N)$ through an estimation of the angular distance as $D_{i}=\left|\theta_{i}-\theta\right|$. The resulting target class is then derived as $\operatorname{argmin} D_{i}$.

\subsection{Multilayer feedforward neural network based on multi-valued neurons}

Neural networks based on complex-valued neurons were reported to learn faster and to generalize better than its traditional counterparts on different benchmarks and real world problems [15, 19, 20]. As was mentioned in [20], the use of complex-valued inputs/outputs, weights and activation functions make it possible to increase the functionality of a single neuron, and of the neural network, by improving the performance and reducing the training time.

We have used a multilayer feedforward neural network based on multi-valued neurons (MLMVN) [15, 19]. Such a network incorporates derivative-free backpropagation training algorithms, resulting in fast convergence to a minimum error [15]. In MLMVN, each neuron, from every hidden and output layer, has connections to all neurons from the previous layer and has a complex activation function $P(z)=z /|z|$, where $z=w_{0}+w_{1} x_{1}+\ldots+w_{n} x_{n}, x_{i} \in \mathbb{C}$ is the output of the $i$-th neuron from previous layer (with $n$ neurons in this layer) and $w_{i} \in \mathbb{C}$ is the corresponding weight.

For our simulations, we have used a MLMVN with 16 inputs, one hidden layer consisting of 24 neurons, and one neuron in the output layer. The network inputs are computed using phase information of the stimulation frequency, and its first harmonic, estimated from all eight electrodes. Considering the EEG data window EEG $(t)=$ $\left(\mathrm{EEG}_{1}(t), \ldots, \mathrm{EEG}_{8}(t)\right)$ of length $T$ seconds $(t \in[0, T])$, the phase $\phi_{k, f}$ of the frequency $f$ from $k$-th channel $\mathrm{EEG}_{k}$ is estimated as $\phi_{k, f}=\arg \left(\int_{0}^{T} \mathrm{EEG}_{k}(t) \cos (2 \pi f t) d t+\right.$ $\left.i \int_{0}^{T} \operatorname{EEG}_{k}(t) \sin (2 \pi f t) d t\right)$. Since the phase information $\phi_{f, k} \in[0,2 \pi)$ (where 0 and $2 \pi$ refer to the same phase), it can be represented by a complex number $e^{i \phi_{k, f}}$ on a unit circle in the complex plane. When estimated in such a way, $16(8 \times 2)$ complex numbers constitute the input $\Phi=\left(e^{i \phi_{1, f}}, \ldots, e^{i \phi_{8, f}}, e^{i \phi_{1,2 f}}, \ldots, e^{i \phi_{8,2 f}}\right)$ to the MLMVN.

The output of the network $\mathcal{N}(\Phi)$ is the index $m(m=$ $1, \ldots, N$, where $N$ is the number of target classes) of the unit circle sector for which $2 \pi(m-1) / N \leq \arg P(z)<$ $2 \pi m / N$. Thus, after the network has been trained, the target classification is obtained by simply looking for a unit circle sector.

\subsection{Classification based on fuzzy logic classifier}

We consider 16 input and 2 output fuzzy system [16], which consists of $N$ fuzzy IF-THEN rules (thus, here, each rule corresponds to the each possible class of phase delayed stimulation) in the form: $R^{i}$ : (IF $\phi_{1, f}$ is $A_{1}^{i}$ AND ... AND IF $\phi_{8,2 f}$ is $A_{16}^{i}$ ) THEN ( $y_{1}$ is $B_{1}^{i}$ AND $y_{2}$ is $B_{2}^{i}$ ), where the fuzzy sets $A_{1}^{i}, \ldots, A_{16}^{i}, B_{1}^{i}, B_{2}^{i}(i=1 \ldots N)$ are characterized by the membership functions $\mu_{A_{j}^{i}}$ and $\mu_{B_{k}^{i}}$, respectively $(j=1 \ldots 16, k=1,2)$. As $\mu_{A_{j}^{i}}$, we use Gaussian functions $\exp \left[-\left(\phi_{k, f}^{i}-\bar{\phi}_{k, f}^{i}\right) / \sigma_{j}^{i}\right]$, where $\bar{\phi}_{k, f}^{i}$ denotes the mean value (assuming circularity of the data) of the phase $\phi_{k, f}$ for frequency $f$ in the $k$-th channel $\mathrm{EEG}_{k}$, for the corresponding phase-shifted class $i$, and $\sigma_{j}^{i}$ is the standard deviation of the data. Since the output classes have to be circular, we divided the unit circle into $N$ equal segments $[2 \pi(m-1) / N, 2 \pi m / N)(m=1, \ldots, N)$, centered at $\varphi_{i}=2 \pi(m-0.5) / N$, and used as the output membership functions $\mu_{B_{k}^{i}} \operatorname{singletons}$ at $\cos \varphi_{i}$ and $\sin \varphi_{i}$, respectively.

The system is based on the Mamdani-type reasoning where antecedents and consequences of each rule are connected by the max t-norm. Fuzzification is done based on the singleton fuzzifier, while defuzzification is based on the center of gravity method. Output crisp values $\bar{y}_{1}$ and $\bar{y}_{2}$ are converted to the target class by finding the $m$ for which $2 \pi(m-1) / N \leq \arg \left(\bar{y}_{1}+i \bar{y}_{2}\right)<2 \pi m / N$.

\section{RESULTS}

As a preliminary step, we have checked the performance of the classifier described in Sec. 3.1, when we use the optimal electrode (as in [13]), and when we use the total set of electrodes, to decide which option is best. The extension to whole set of electrodes was done by searching for the target 


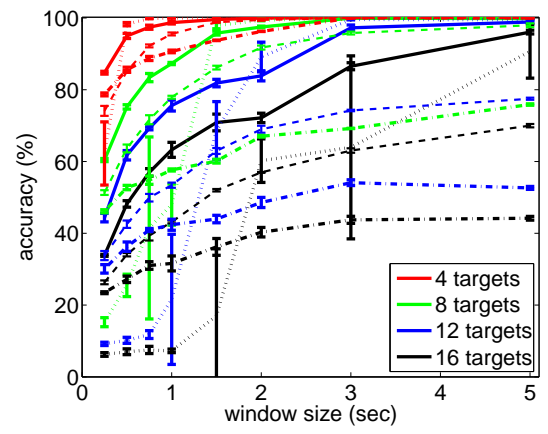

(a)

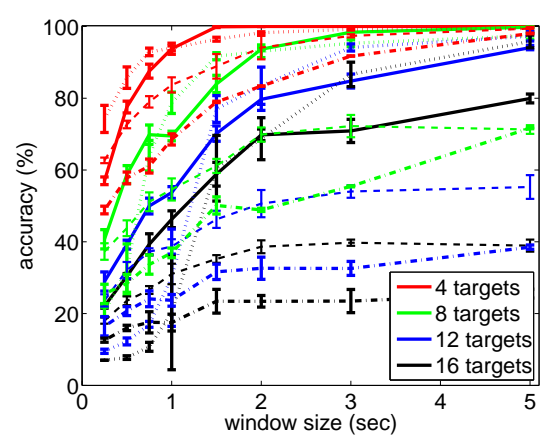

(d)

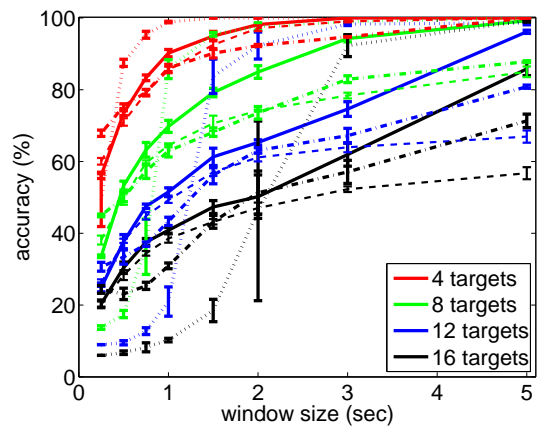

(b)

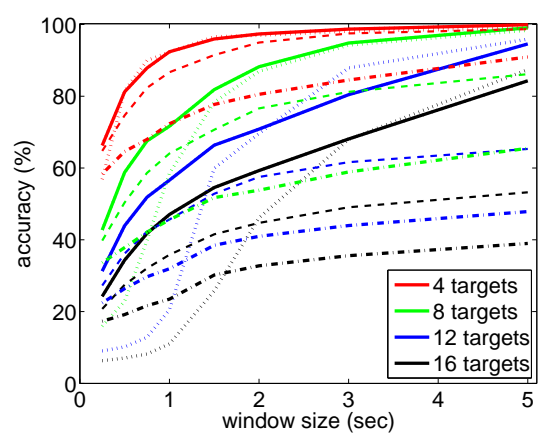

(e)

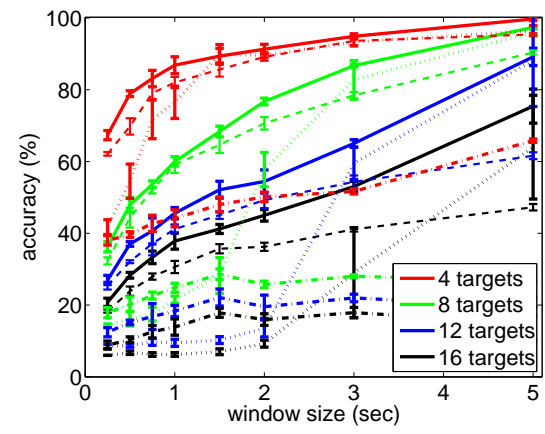

(c)

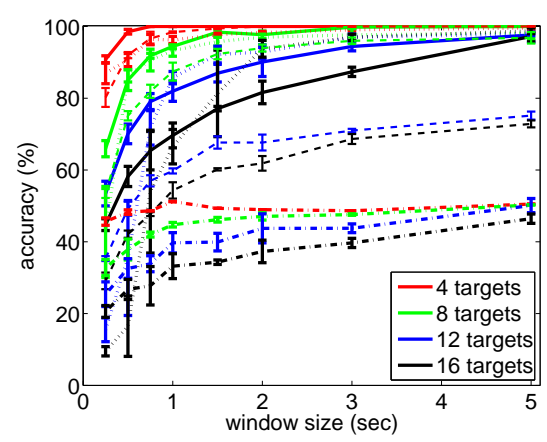

(f)

Fig. 1: (a-d) Decoding results for 4 subjects. The results are shown for 4, 8, 12, and 16 targets (phase shifts) and plotted as a function of window size. The stimulation frequency is $f=85 / 6 \mathrm{~Hz}$. The error bars indicate standard deviations. (e) Decoding results averaged over all subjects. The stimulation frequency is $f=85 / 6 \mathrm{~Hz}$. (f) Decoding results for the same subjects as in (c) but for the stimulation frequency $f=85 / 10=8.5 \mathrm{~Hz}$. The results are shown for $4,8,12$, and 16 targets (phase shifts). The classifiers considered are: the one taken from [14] (dash-dot lines), an extended version of the one taken from [13] (dashed lines), the MLMVN (dotted lines), and the one based on fuzzy logic (solid line).

class that maximizes the sum of the projections into reference phases, for all electrodes, for the fundamental- and the first harmonic frequencies. Our results suggest that, in general, considering all channels gives superior results. This setting was used for comparing the different decoding algorithms.

We have also checked the performance based on electrode $\mathrm{Oz}$ (the same electrode as in [14]) of the classifier described in Sec. 3.2, when we use $1 \mathrm{~min}$. of EEG recording for deriving $t_{\text {ref }}$, as in [14] (for this we used a 2-fold cross-validation on our 2 min. data), and when we consider above described 4fold approach. The obtained results did not reveal any big differences. Thus, we opted for the 4-fold approach when comparing all decoding algorithms.

The comparison is shown in Fig. 1(a-d), for our four subjects individually, for stimulation frequency $f=85 / 6 \mathrm{~Hz}$, and for different numbers $N$ of target classes; the results averaged among all subject performance is shown in Fig. 1(e).

\section{DISCUSSION}

As it can be seen from Fig. 1(c), the performance of one of the subjects is quite low. This could be due to the fact that this subject did not generate a proper SSVEP at the chosen frequency $(85 / 6 \mathrm{~Hz})$. To validate this, we made a second test with this subject, but at a lower frequency $(8.5 \mathrm{~Hz})$. As we can see from Fig. 1(f) the accuracy improves. This suggests that the stimulation frequency is subject dependent and should be selected preferably during a calibration stage.

Among all considered classifiers, the one based on fuzzy logic yields, in general, better results for any number of targets and any window size. Based on this, we recommend the fuzzy approach for on-line brain-computer interface (BCI) based on phase-shifted SSVEP decoding. In addition, it has the advantage that we do not need to train it, contrary to, e.g., the MLMVN.

Concerning our second proposed classifier (MLMVN), we can see that it achieves a good classification performance for all subjects, for a suitable window length. But the ac- 
curacy strongly depends on the number of targets: a good performance is achieved starting from $T=1-1.5$ second window size for four targets, 2-3 second window for eight targets, above 3 second window for 12 targets, and so on. But for smaller window sizes, the algorithm of [13] yields better results than the MLMVN, but the obtained accuracy falls short. The inferior performance of the MLMVN for small window sizes could be explained by the fact that we do not have a clearly separable training set, which is attempted to be fitted by the nonlinear MLMVN classifier. Thus, while fitting a badly separable training set, the MLMVN is not able to generalize properly (and we have a performance close to chance level). While for more separable data sets (the case of a larger window size $T$ ), the MLMVN is able to generalize much better than, for example, the classifier used in [13].

Our results suggests that the classifier proposed in [14] has the lowest performance. This can be explained by the fact that such a classifier relies only on one channel and on only the fundamental frequency while other classifiers benefit from additional information obtained from other channels and the first harmonic. It also should be noted that, in our comparison, this classifier performed worse than mentioned in the original paper [14]. This can be due to the following: we use a CRT monitor for visual stimulation, instead of an LED (light-emitting diode), which could lead to inferior SSVEP responses; our stimulation frequency is more than twice lower than in [14], which results in a twice larger time interval to arrive at the same number of stimulation periods for averaging; the interval for band-pass filtering can be frequency dependent (not $[f-2, f+2] \mathrm{Hz}$, as we took here). Additionally to this, we should mention that the sampling rate in the system proposed in [14] was 8 times higher than in our EEG device, which gives a better accuracy of the method from Sec. 3.2, due to rounding errors.

In addition, the comparison results suggest that, for a small amount of target classes (4 classes), all classifiers produce almost equal results. But when considering eight target classes or more, we can see a differentiation in the classifiers' accuracies: the gain in accuracy for the MLMVN and the classifier based on fuzzy logic becomes more prominent.

For future steps, additionally to the phase information from each channel separatelly, we can also include features showing interchannel relation, such as synchronization [21] and characteristics of propogating waves [22]. We should also consider the construction of an on-line BCI system and its evaluation on larger subject group. For these purposes, a search for a proper subset of electrodes is also highly advisable (without significant loss in accuracy), as it increases the user-friendliness of the system.

\section{CONCLUSION}

We have compared the performance of different classifiers decoding multi-stimulus/multi-target responses in SSVEP BCI, when the targets are encoded by phase shifts. The classifier based on fuzzy logic was shown to possess a superior performance, compared to the other classifiers considered, recommending its use for with on-line phase-based SSVEP decoding.

\section{REFERENCES}

[1] P. Sajda, K.-R. Müller, and K.V. Shenoy, "BrainComputer Interfaces," IEEE Signal Proc Magazine, vol. 25 , no. 1 , pp. 16-17, 2008.

[2] M. Velliste, S. Perel, M.C. Spalding, A.S. Whitford, and A.B. Schwartz, "Cortical control of a prosthetic arm for self-feeding," Nature, , no. 453, pp. 1098-1101, 2008.

[3] N.V Manyakov and M.M.. Van Hulle, "Decoding grating orientation from microelectrode array recordings in monkey cortical area V4," International Journal of Neural Systems, vol. 20, no. 2, pp. 95-108, 2010.

[4] M.A. Lebedev and M.A.L. Nicolelis, "Brain-machine interface: past, present and future," Trends in Neurosc, vol. 29, no. 9, pp. 536-546, 2005.

[5] N. Birbaumer, A. Kübler, N. Ghanayim, T. Hinterberger, J. Perelmouter, J. Kaiser, B. Iversen, I.and Kotchoubey, N. Neumann, and H Flor, "The thought translation device (TTD) for completely paralyzed patients," IEEE Transactions on Rehabilitation Egineering, vol. 8, no. 2, pp. 190-193, 2000.

[6] B. Blankertz, G. Dornhege, M. Krauledat, K.-R. Müller, and G. Curio, "The non-invasive Berlin brain-computer interface: fast acquisition of effective performnce in untrained subjects," Neuroimage, vol. 37, no. 2, pp. 539$550,2007$.

[7] C. S. Herrmann, "Human EEG Responses to 1-100 Hz Flicker: Resonance Phenomena in Visual Cortex and Their Potential Correlation to Cognitive Phenomena," Exp. Brain Res., vol. 137, pp. 346-353, 2001.

[8] M. Cheng, X. Gao, S. Gao, and D. Xu, "Design and Implementation of a Brain-Computer Interface with High Transfer Rates," IEEE Transactions on Biomedical Engineering, vol. 49, no. 10, pp. 1181-1186, 2002.

[9] Y. Gao, Wang. R., Gao X., Hong B., and Gao S., "A Practical VEP-based Brain-Computer Interface," IEEE Transactions on Neural Systems and Rehabilitation Engineering, vol. 14, no. 2, pp. 234-240, 2006.

[10] N.V. Manyakov, N. Chumerin, A. Combaz, A. Robben, and M.M. Van Hulle, "Decoding SSVEP Responses Using Time Domain Classification," in Proc. of the International Conference on Fuzzy Computation and 2 nd In- 
ternational Conference on Neural Computation, 2010, pp. 376-380.

[11] B. Allison, T. Luth, D. Valbuena, A. Teymourian, I. Volosyak, and A. Gräser, "BCI Demographics: How Many (and What Kinds of) People Can Use an SSVEP BCI?," IEEE Transactions on Neural Systems and Rehabilitation Engineering, vol. 18, no. 2, pp. 107-116, 2010.

[12] I. Volosyak, H. Cecotti, and A. Gräser, "Impact of Frequency Selection on LCD Screens for SSVEP Based Brain-Computer Interface," in Proc. IWANN, Part I, LNCS 5517, 2009, pp. 706-713.

[13] C. Jia, X. Gao, B. Hong, and S. Gao, "Frequency and Phase Mixed Coding in SSVEP-based Brain-Computer Interface," IEEE Transaction on Biomedical Engineering, vol. 58, no. 1, pp. 200-206, 2011.

[14] P-L. Lee, J-J. Sie, Y-J. Liu, C-H. Wu, M-H. Lee, C-H. Shu, P-H. Li, C-W. Sun, and Shyu K-K., "An SSVEPActuated Brain Computer Interface Using Phase-Tagged Flickering Sequences: A Cursor System," Annals of Biomedical Engineering, vol. 38, no. 7, pp. 2383-2397, 2010.

[15] I. Aizenberg and C. Moraga, "Multilayer Feedforward Neural Network Based on Multi-valued Neurons (MLMVN) and a Backpropagation Learning Algorithm," Soft Comput, vol. 11, pp. 169-183, 2007.

[16] L. Rutkowski, Computational intelligence: methods and techniques, Springer Verlag, 2008.

[17] R.F. Yazicioglu, T. Torfs, P. Merken, J. Penders, V. Leonov, R. Puers, B. Gyselinckx, and C. Van Hoof, "Ultra-low-power Biopotential Interfaces and Their Applications in Wearable and Implantable Systems," $M i$ croel J, vol. 40, no. 9, pp. 1313-1321, 2006.

[18] H. Strasburger, "The Analysis of Steady State Evoked Potentials Revised," Clin Vis Sci, vol. 1, no. 3, pp. 245256, 1987.

[19] I. Aizenberg, D. Paliy, J.M. Zurada, and J. Astola, "Blur Identification by Multilayer Neural Network based on Multi-Valued Neurons," IEEE Transactions on Neural Networks, vol. 19, no. 5, pp. 883-898, 2008.

[20] I. Aizenberg, "Complex-Valued Neurons with PhaseDependent Activation Functions," in ICAISC 2010, Part II, LNAI 6114, 2010, pp. 3-10.

[21] N.V. Manyakov and M.M. Van Hulle, "Synchronization in monkey visual cortex analyzed with an informationtheoretic measure," Chaos, vol. 18, no. 3, pp. 037130 , 2008.
[22] N.V. Manyakov, R. Vogels, and M.M. Van Hulle, "Decoding stimulus-reward pairing from local field potentials recorded from monkey visual cortex," IEEE Transactions on Neural Networks, vol. 21, no. 12, pp. 18921902, 2010. 